\title{
A Robust Image Watermarking Scheme Based on the Laplacian Pyramid Transform
}

\author{
Nguyen Chi Sy, Ha Hoang Kha \\ Faculty of Electrical \& Electronics Engineering, Ho Chi Minh City University of Technology, VNU-HCM, Vietnam \\ E-mail: chisy.nguyen@gmail.com,hhkha@hcmut.edu.vn
}

Nguyen Minh Hoang

Saigon Institute of Information Communication Technology

E-mail: nmhoang@gmail.com

Keywords: robust watermarking, Laplacian pyramid, framing pyramids

Received: November 20, 2017

\begin{abstract}
This paper is concerned with the digital image watermarking techniques to protect intellectual property and to authenticate digital images. Different from the most conventional methods using the discrete cosine transforms (DCT) and discrete-wavelet transforms (DWTs), this paper exploits the improved Laplacian pyramid transform to develop a new image watermarking scheme in which the improved Laplacian pyramid transform is used to decompose and reconstruct the host image. Then, to select an appropriate watermarking solution, we investigate the various frequency sub-band regions with different the levels and strength factors to perform the watermark embedding. Finally, we conduct experiments to investigate the invisibility and robustness of the proposed algorithm in terms the peak signal-to-noise ratio (PSNR), normalized correlation (NC), and structural similarity index (SSIM). Experimental results showed that our proposed scheme offers good robustness and invisibility. As compared to the watermarking schemes using the curvelets, our watermarking scheme is more robust for the lossy JPEG compression and Gaussian low pass filtering attacks. In addition, our method is also efficient in terms of computational time.
\end{abstract}

Povzetek: Članek predstavlja novo obliko zaščite slik s pomočjo Laplacove piramidne transformacije.

\section{Introduction}

Since the rapid development of communication networks and advances in digital signal processing have lead to the multimedia piracy issues, copyright protection of multimedia products has become an extensive research topic. To protect content of the multimedia data from the modification and to provide content authentication, watermarking methods have been used [1, 2, 3]. The watermarking method is to embed or hide digital information, known as watermark, into a multimedia product. Then, one can extract the watermark data when necessary for verifying the authenticity or the integrity of the carrier signals, identifying its owners, or tracing copyright infringements. The digital watermarking schemes can be applied to various digital multimedia data such as audio, image and video. In this paper, we focus on the digital watermarking for digital image.

\subsection{Related works}

Watermarking methods for digital images can be implemented in spatial domain or in transform domains. Watermarking schemes in spatial domain directly modify the gray level values of pixels. It has been known that the watermarking methods in spatial domain are ineffective since the watermarks can be easily destroyed by common sig- nal processing operations [4]. To overcome this drawback, transform domain based watermarking schemes have been actively studied [5]. With regards to transform domain based watermarking schemes, two typical transforms that have been widely used are discrete cosine transform (DCT) and discrete wavelet transform (DWT) (see, for example, $[3,6,7,8,9]$, and references therein). In general, the desired properties of watermarking schemes are the robustness, the invisibility and the capacity [10]. However, there are tradeoffs between these desirable properties. Reference [3] showed that the DCT based watermarking techniques are superior to ones based on spatial domain in terms of robustness. In addition, reference [6] demonstrated that DCT based watermarking schemes are robust against such the common signal processing attacks as low-pass filtering, reducing image quality (blurring), and adjusting contrast and brightness. However, DCT based watermarking techniques are unsustainable with the geometric transform attacks, for example, rotation, rescaling, and cutting operations [9]. A1ternatively, by using the wavelet transform into watermarking schemes, the authors in [3] showed that watermarking schemes based on wavelet transform outperform those on DCT approaches. It should be noted that in compression and denoising applications, the coefficients in the transform domain are quantized or performed thresholding operations and, thus, there exist errors in reconstructed images. 
Recently, the directional transforms have been exploited in the watermarking schemes. In [11], the authors introduced a digital image watermarking scheme using the curvelet transform domain. By using the scale distribution, Human Visual System (HVS) and curvelet coefficients, they selected the appropriate positions to insert the watermark. In their method, the binary watermark of $21 \times 21$ was used. By experimental results, they showed that the embedding watermark in the curvelet domain ensures robustness and invisibility. In addition, they also indicated that watermarking in the curvelet domain offers the improved robustness and invisibility as compared to those in the ridgelet domain.

On the other hand, reference [12] proposed a digital image watermarking algorithm operating in the fast curvelet transform in which they selected the medium frequency coefficients to embed the binary watermark image of $32 \times 32$ pixels. In [12], the authors illustrated that their proposed watermarking scheme is good at both invisibility and security. In addition, experiment results therein showed that their watermarking scheme offers good robustness against noise, cropping, filtering, JPEG compression and other attacks. Reference [13] proposed a blind watermarking based on the curvelet transform domain. In order to achieve both invisibility and robustness, many different scales of curvelet transform domain have been investigated to choose the appropriate scales to embed the watermark. Experimental results showed the advantages of their method as compared to a watermarking scheme in the DCT-DWT combined domain for the lossy JPEG compression attacks, speckle and Gaussian noise. Alternatively, the authors in [14] proposed Laplacian pyramid (LP) scheme to represent multi-resolution for images. The advantages of the LP scheme are its simplicity and low computation complexity. However, there exists some drawbacks in the LP schemes, such as implicit oversampling [15]. The authors in [15] proposed an improved Laplacian pyramid (LP) scheme by exploiting an efficient filter bank (FB). This approach is proved to be more efficient than the conventional methods for the signal reconstruction degraded by noise.

\subsection{Motivation and contributions}

Motivated from the advantages of an improved Laplacian pyramid (LP) scheme in [15] and inspired by the works in $[11,12,16]$, we develop a blind watermarking algorithm in improved LP domain in which the symmetric bi-orthogonal and the new reconstruction methods are used. More specifically, we propose a blind watermarking using the improved Laplacian Pyramid transform. To balance the invisibility and the robustness, we exploit the low frequency and the mid frequency regions to embed the watermark. We investigate the various levels and strength factors to choose the appropriate values. The watermark is a binary image whose size is $32 \times 32$. To evaluate the performance of the watermarking schemes, we use the performance met- rics as the peak signal-to-noise ratio (PSNR), normalized correlation (NC) and the structural similarity index (SSIM) to measure the invisibility and the robustness of the algorithms. Our experimental results showed that the proposed watermarking scheme offers high invisibility and robustness. As compared to the watermarking schemes based on curvelets, the proposed algorithm has better invisibility and robustness for the lossy JPEG compression attack.

The rest of the paper is organized as follows. In Section 2, we introduce a proposed watermarking scheme in which the improved Laplacian pyramid and a new reconstruction using projection are used. Then, watermark embedding and extracting schemes with selective levels and strength factors are introduced as well. Section 3 presents experimental results and discussions. Finally, the concluding remarks are presented in Section 4.

The contributions in this paper have been partly presented in the 2017 International Conference on Recent Advances in Signal Processing, Telecommunications \& Computing [17].

\section{Proposed watermarking scheme using Laplacian pyramid transform}

In this section, we present a blind watermarking scheme. The embedding and extracting watermark algorithms are shown in Figure 1 and Figure 2, respectively. In our blind watermarking scheme, the host image is firstly analyzed into improved LP coefficients by Laplacian pyramid toolbox. We present the improved LP transform in detail in 2.1. To enhance the security for the watermark, we use the Arnold transform on the watermarks which shall be described in 2.2. The embedding scheme in detail is explained in 2.3 and the extracting scheme in detail is described in 2.4 .

\subsection{Laplacian pyramid and novel reconstruction method}

\subsubsection{Burt and Adelson's Laplacian Pyramid}

The block diagram for analysis and synthesis of the LP is shown in Figure 3 in which $\mathbf{x}$ is the input signal, output $\mathbf{c}$ is a coarse approximation while output $\mathbf{d}$ is a difference between the original signal and the prediction $\mathbf{p}[14,15]$. First, using low-pass filtering and down sampling yields a coarse approximation of the original. The coarse approximation signal is given by

$$
c[n]=\sum_{k \in Z^{d}} x[k] h[M n-k]=\langle x, \widetilde{h}[.-M n]\rangle
$$

where $n, k \in Z^{d}, h[n]=h[-n]$. The coarse components are up-sampled and filtered to yield the prediction compo- 
nent which is given by

$$
p[n]=\sum_{k \in Z^{d}} \mathrm{c}[k] \mathrm{g}[n-M k] .
$$

In terms of matrices and vectors, the coarse and prediction components are expressed as $\mathbf{c}=H \mathbf{x}$ and $\mathbf{p}=G \mathbf{c}$ where $\mathbf{x}=\left(x[n]: n \in Z^{d}\right), G$ and $H$ correspond to $G(\uparrow M)$ and $H(\downarrow M)$, respectively. Then, the difference between the

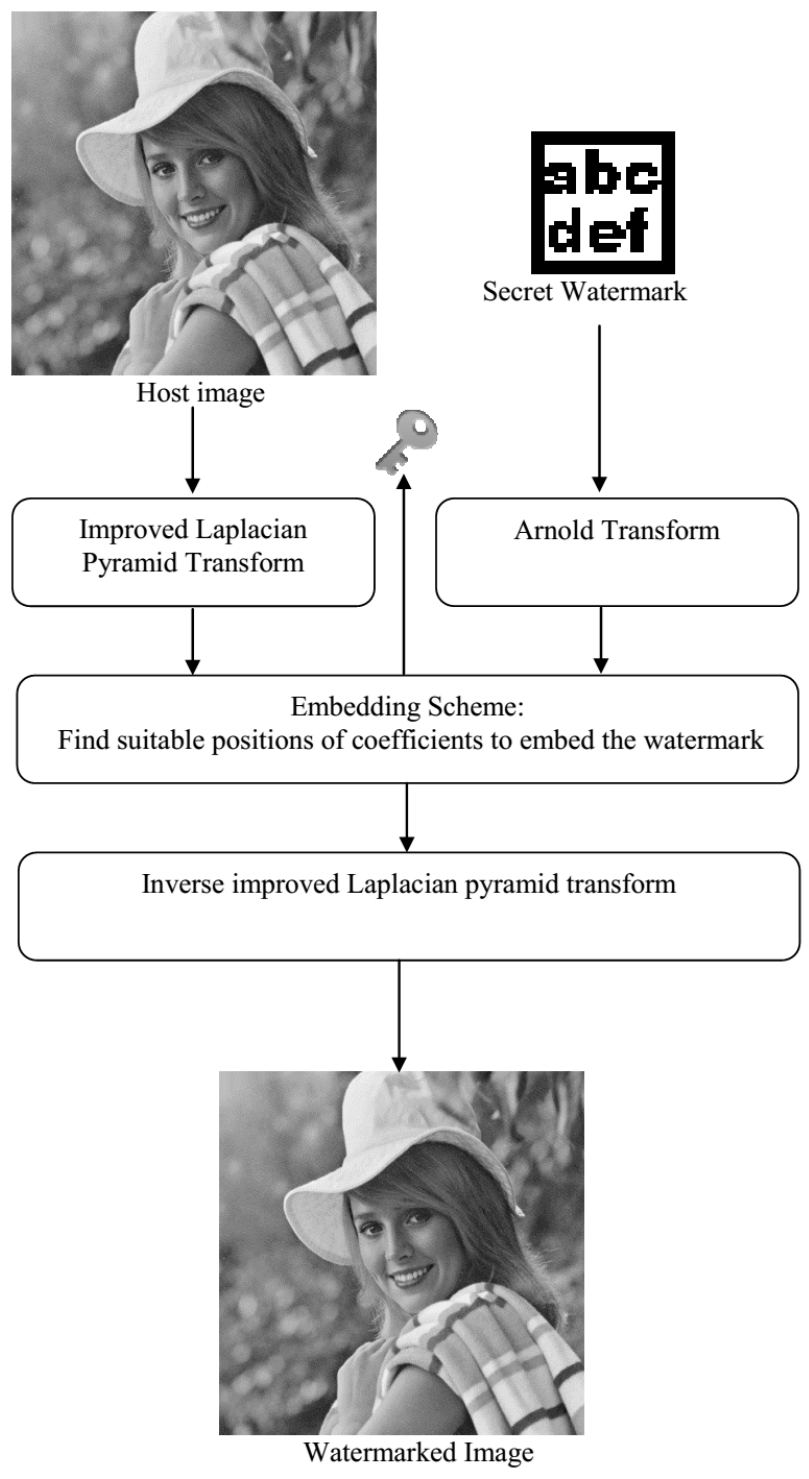

Figure 1: The proposed watermark embedding scheme

original signal and this predicted counterpart, known as the prediction error, is defined by

$$
\mathbf{d}=\mathbf{x}-\mathbf{p}=\mathbf{x}-G H \mathbf{x}=(I-G H) \mathbf{x} .
$$

Accordingly, we can rewrite the analysis operator of LP as

$$
\left(\begin{array}{l}
\mathbf{c} \\
\mathbf{d}
\end{array}\right)=\left(\begin{array}{l}
H \\
I-G H
\end{array}\right) \mathbf{x} .
$$

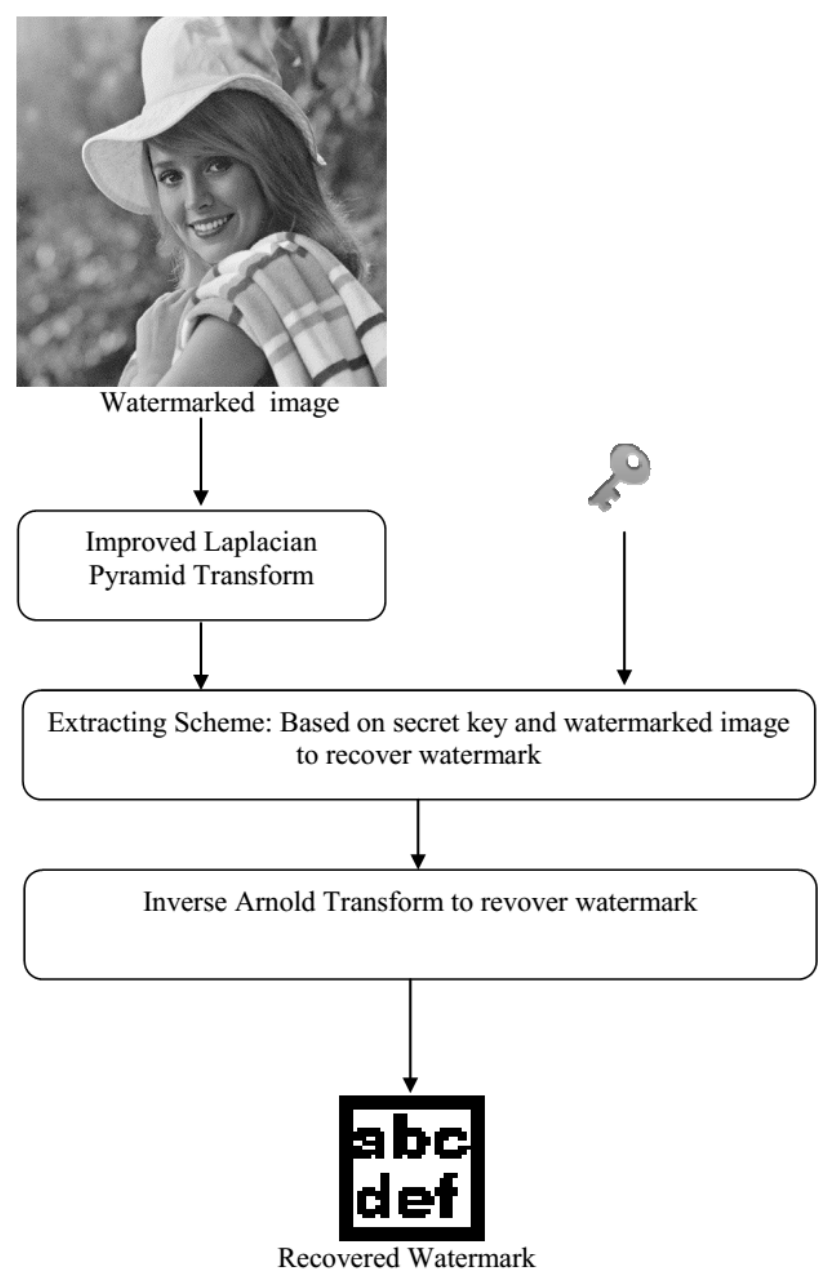

Figure 2: The proposed watermark extracting scheme.

The inverse transform of the LP is shown Figure 3(b) in which $\hat{\mathbf{x}}=G \mathbf{c}+\mathbf{d}$ and, thus, one has

$$
\hat{\mathbf{x}}=\left(\begin{array}{ll}
G & I
\end{array}\right)\left(\begin{array}{l}
\mathbf{c} \\
\mathbf{d}
\end{array}\right) .
$$

It has been shown in [15] that LP can be perfectly reconstructed with any pair of filters $H$ and $G$.

\subsubsection{Reconstruction using projection}

A new reconstruction method is shown in Figure 4 [15]. From Figure 4, the improved inverse transform of LP can be written as

$$
\hat{\mathbf{x}}=\left(\begin{array}{cc}
G & I-G H
\end{array}\right)\left(\begin{array}{l}
\mathbf{c} \\
\mathbf{d}
\end{array}\right) .
$$

Let $S_{2}=\left(\begin{array}{cc}G & I-G H\end{array}\right)$ be a transform matrix for the reconstruction algorithm. From Equations (4) and (6), we have $S_{2} A=I-G H+(G H)^{2}$. Thus, $S_{2}$ is a left inverse of $A$ if and only if $G H=(G H)^{2}$, i.e., $G H$ is a projector. The projection condition is

$$
H G=I
$$




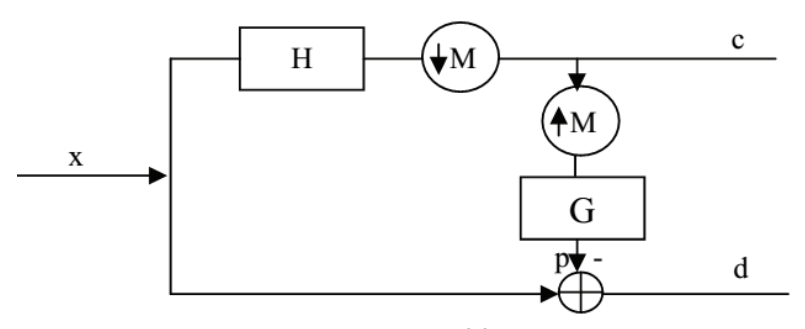

(a)

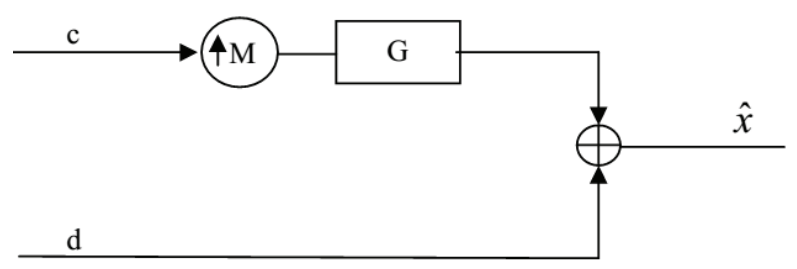

(b)

Figure 3: The typical Laplacian pyramid transform: Analysis scheme: (b) Synthesis diagram.

or

$$
\langle\widetilde{h}[.-M k], g[.-M l]\rangle=\delta[k-l] \forall k, l \in Z^{d} .
$$

Any filters $\mathrm{H}$ and $\mathrm{G}$ are called bi-orthogonal filters if they satisfy condition (8). The reconstruction scheme in Figure 4 is equivalent to an inverse transform of the LP if and only if two filters $H$ and $G$ are bi-orthogonal with given sampling lattice $M$. That is, the prediction operator of the $L P(G H)$ is a projector. In this paper, we use the $9-7$ biorthogonal filters whose coefficients are shown in Table 1. It is important to evaluate the reconstruction performance of the two methods in Figure 3(b) (namely, REC-1) and Figure 4 (REC-2). Suppose that one wants to approximate $\mathbf{x}$ given $\hat{\mathbf{y}}=\mathrm{A} \mathbf{x}+\boldsymbol{\eta}$. Without information about the error $\boldsymbol{\eta}$, $\hat{\mathbf{x}}$ is chosen such that the residual $\|\mathrm{A} \hat{\mathbf{x}}-\hat{\mathbf{y}}\|$ is minimized. Using this measurement to evaluate the reconstruction performance, reference [15] showed that $R E C-2$ outperforms $R E C-1$.

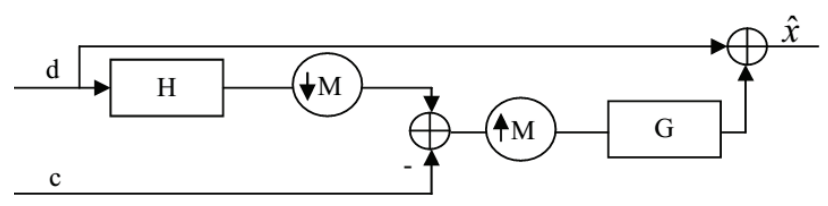

Figure 4: New reconstruction diagram for the LP scheme [14].

\subsection{The Arnold transform}

To provide a improved security for the watermark, the Arnold transform is adopted to make the watermark uncertain. With the Arnold transform, the watermark cannot be defined even when it is detected. This transform also improves the robustness of the watermark. The Arnold transform function is given by [12]

$$
\left(\begin{array}{l}
x^{\prime} \\
y^{\prime}
\end{array}\right)=\left(\begin{array}{ll}
1 & 1 \\
1 & 2
\end{array}\right)\left(\begin{array}{l}
x \\
y
\end{array}\right) \bmod N
$$

where $N$ is the watermark image size and the point $\left(x^{\prime}, y^{\prime}\right)$ is a shifted version of point $(x, y)$.

\subsection{The watermark embedding scheme}

To embed the watermark into the LP transform domain, the improved LP decomposes the host image into multiscale images. Since the Human Vision System (HVS) is very sensible to the low frequency coefficients, the watermark should be embedded into the high frequency coefficients in order to increase invisibility. However, the common image processing attacks normally affect the high frequencies of the image signals. Thus, robustness is improved if the watermark is inserted into the low frequencies. It is worth noting that robustness plays an important role for applications of protecting digital image copyrights. Thus, to increase the embedded watermark size and to enhance the robustness of the proposed algorithm, we have investigated the embedding of watermark into the prediction error coefficients $\mathbf{d}$ at both low and middle frequencies from level number $5\left(\mathbf{d}_{5}\right)$ (from low frequency to high frequency: 5,4,3,2,1) as shown in Figure 5. The scheme for embedding watermark is described in detail in Algorithm 2 in which $\ell$ is the decomposed level; $\mathbf{d}_{\ell}$ is image of the prediction error at level $\ell ; p_{1}$ and $p_{2}$ are position parameters of $\mathbf{d}_{l} ; k$ is position parameter of watermark. Function move $N \operatorname{ext}\left(d\left(\ell, p_{1}, p_{2}\right)\right)$ returns the next coefficient of $d\left(\ell, p_{1}, p_{2}\right), \beta$ is a metric of embedment strength, $\max \left(\mathbf{d}_{\ell}\right)$ is the largest coefficient of prediction error of level $\ell$. Each bit of binary watermark is embedded in an improved LP coefficient. This coefficient is determined as follows. In each level $(\ell)$ selected to embed the watermark, we calculate a threshold value $T_{\ell}=\beta \times \max \left(\mathbf{d}_{\ell}\right)$. The value of $T_{\ell}$ affects on the invisibility and robustness of watermarking schemes [11]. If the value of $T_{\ell}$ is large, robustness will be strong, vice versa. The value of $\beta$ belongs to $0<\beta \leq 1$. Because the low frequency coefficients are used to embed the watermark in the proposed method, to balance between the invisibility and the robustness, the value of $\beta$ is set to 0.2 [16] for all levels. The selected coefficient to embed a bit of watermark depends on the value of embedded bit and the value of examining coefficient as compared with $T_{\ell}$. If it is not the case where the coefficient do not satisfies the predefined conditions, the next coefficient will be considered. The positions of the selected coefficients are recorded in order to reuse in the watermark extracting scheme. The watermark embedding scheme is summarized in Algorithm 2. 
Table 1: The $9-7$ bi-orthogonal filters with coefficients.

\begin{tabular}{|c|c|c|c|c|c|}
\hline$n$ & 0 & \pm 1 & \pm 2 & \pm 3 & \pm 4 \\
\hline$h[n]$ & 0.852699 & 0.377403 & -0.110624 & -0.023894 & 0.037828 \\
\hline$g[n]$ & 0.788486 & 0.418092 & -0.040689 & -0.064539 & \\
\hline
\end{tabular}

\subsection{An algorithm for extracting watermarks}

To extract the watermarks, the watermarked image firstly is transformed into the improved LP domain. Based on the information recorded in Algorithm 2 about the positions selected to embed the binary watermark, we calculate and determine whether the bit at this position is bit 0 or bit 1 . Second, as similar as in the scheme for embedding watermark, the threshold value is calculated by Equation (11). Third, we obtain sequently the positions recorded in the scheme for embedding watermark to seek the coefficient selected to embed a bit watermark. Finally, each coefficient will be processed and compared to the parameter $T_{\ell}{ }^{\prime}$ to decide whether the bit embedded in this coefficient is bit 0 or bit 1 . The description in detail of the extracting algorithm is represented in Algorithm 3 in which $\ell$ is the decomposed level; $\mathbf{d}_{\ell}{ }^{\prime}$ is image of the prediction error at level $\ell ; p_{1}$ and $p_{2}$ are position parameters of $\mathbf{d}_{\ell} ; k$ is position parameter of watermark.

\subsection{Performance metrics of an image watermarking algorithm}

To measure the invisibility and robustness of the watermarking scheme, four parameters, namely the ratio of peak signal to noise (PSNR), the normalized correlation (NC), the structural similarity index (SSIM) and the execution time for embedding watermark and extracting watermark are typically considered $[11,12]$. To assess the difference between the original image and the processed or attacked one, we can use three first metrics. Assume that the dimension of the images is $M \times N$ and the pixels of the original image and of the watermarked images are $X_{i j}$ and $W_{i j}$, respectively. To measure the invisibility between the original gray image and the watermarked one, we can use the PSNR defined by

$$
P S N R=10 \log _{10} \frac{(255)^{2}}{M S E} d B
$$

where the mean square error (MSE) is given by

$$
M S E=\frac{1}{M * N} \sum_{i=1}^{M} \sum_{j=1}^{N}\left(X_{i j}-\mathrm{W}_{i j}\right)^{2}
$$

On the other hand, the NC can measure the difference between original watermark and extracted watermark. Thus, to assess the robustness between the original watermark
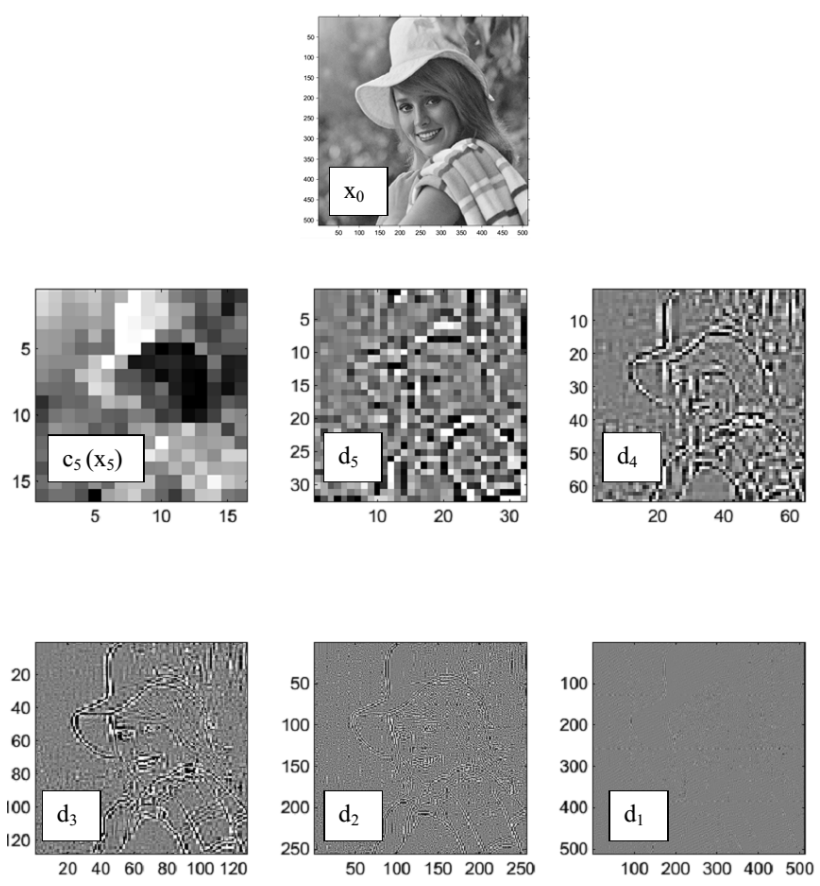

Figure 5: The results of decomposition host image by using the improved LP transform with 5 levels, and images of improved LP.

and recovered watermark, the NC can be used. The formula of NC is defined by

$$
N C=\frac{\sum_{i=1}^{M} \sum_{j=1}^{N} W_{o i j} W_{r i j}}{\sqrt{\sum_{i=1}^{M} \sum_{j=1}^{N} W_{o i j}^{2}} \sqrt{\sum_{i=1}^{M} \sum_{j=1}^{N} W_{r i j}^{2}}}
$$

where the pixels of the original watermark and the extracted watermark image of $M \times N$ dimension are $W_{o i j}$ and $W_{\text {rij }}$, respectively. It is obvious that $\mathrm{NC}$ has the values from 0 to 1 , and $\mathrm{NC}$ value of 1 reveals the best robustness. The robustness of watermarking schemes is also reflected by the NC when watermarked image is attacked on.

Structural Similarity index (SSIM) is commonly used to measure the similarity between two images [18]. Three components including luminance, contrast and structures are compared to compute SSIM. The value of SSIM is between 0 and 1 , where 1 means two image identical and 0 means two image totally different. At each step, the local window is used to calculate the local statistics and SSIM index. Final local SSIM measure is the product of three 


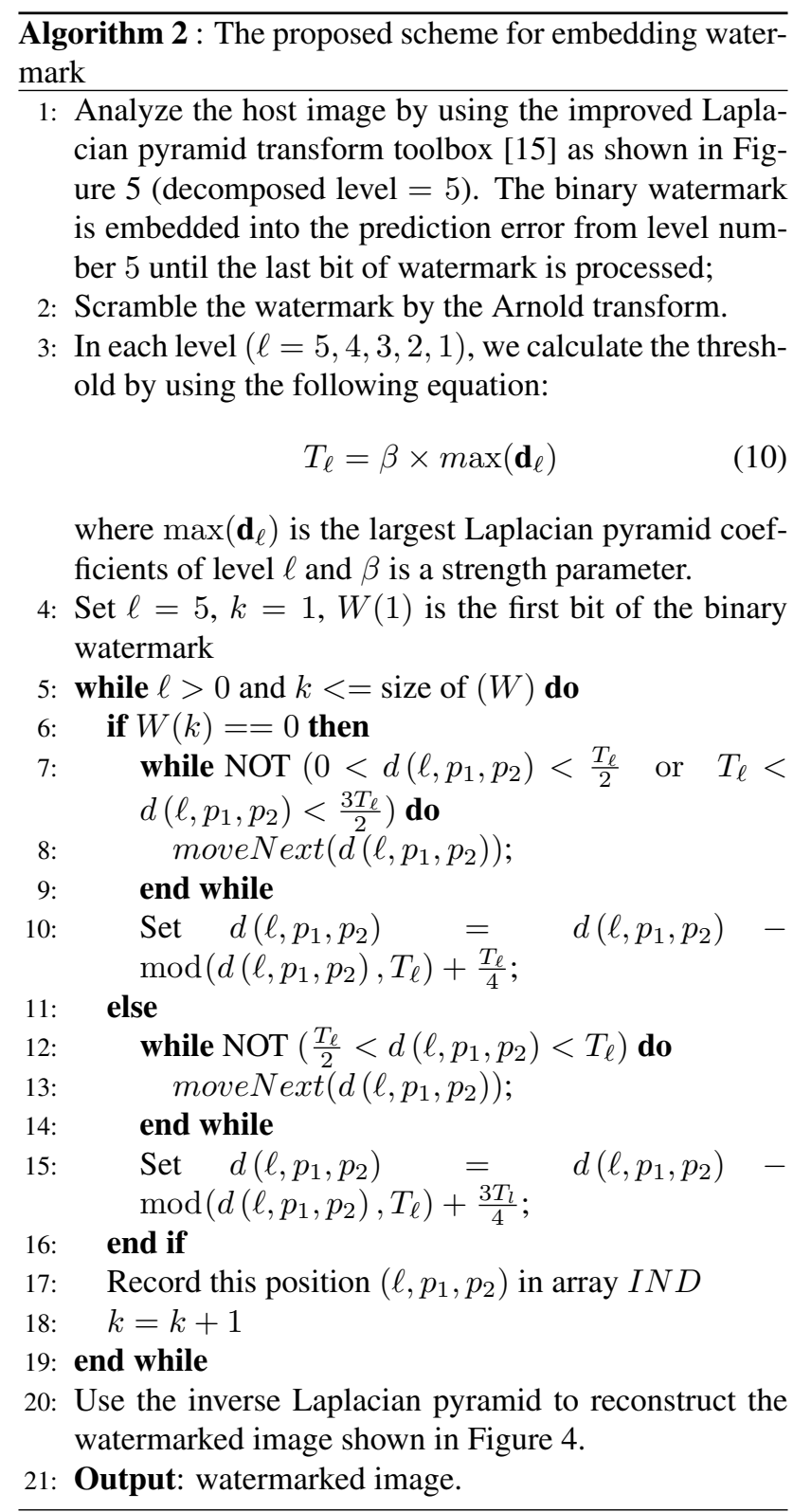

components: luminance, contrast and structure as follows:

$$
S S I M=\frac{\left(2 . \gamma_{x} \cdot \gamma_{y}+C_{1}\right)\left(2 . \rho_{x y}+C_{2}\right)}{\left(\gamma_{x}^{2}+\gamma_{y}^{2}+C_{1}\right)\left(\rho_{x}^{2}+\rho_{y}^{2}+C_{2}\right)}
$$

where:

- $\gamma_{x}$ and $\gamma_{y}$ are weighted means from original and degraded image,

- $\rho_{x}$ and $\rho_{y}$ are weighted variances from original and degraded image.

- $\rho_{x y}$ is similarly defined as weighted covariance between original and degraded image.

- $C_{1}$ and $C_{2}$ are constants.

Another metric used to evaluate our proposed method is execution time which is the amount of time required to embed a watermark into the host image, and then extract it.

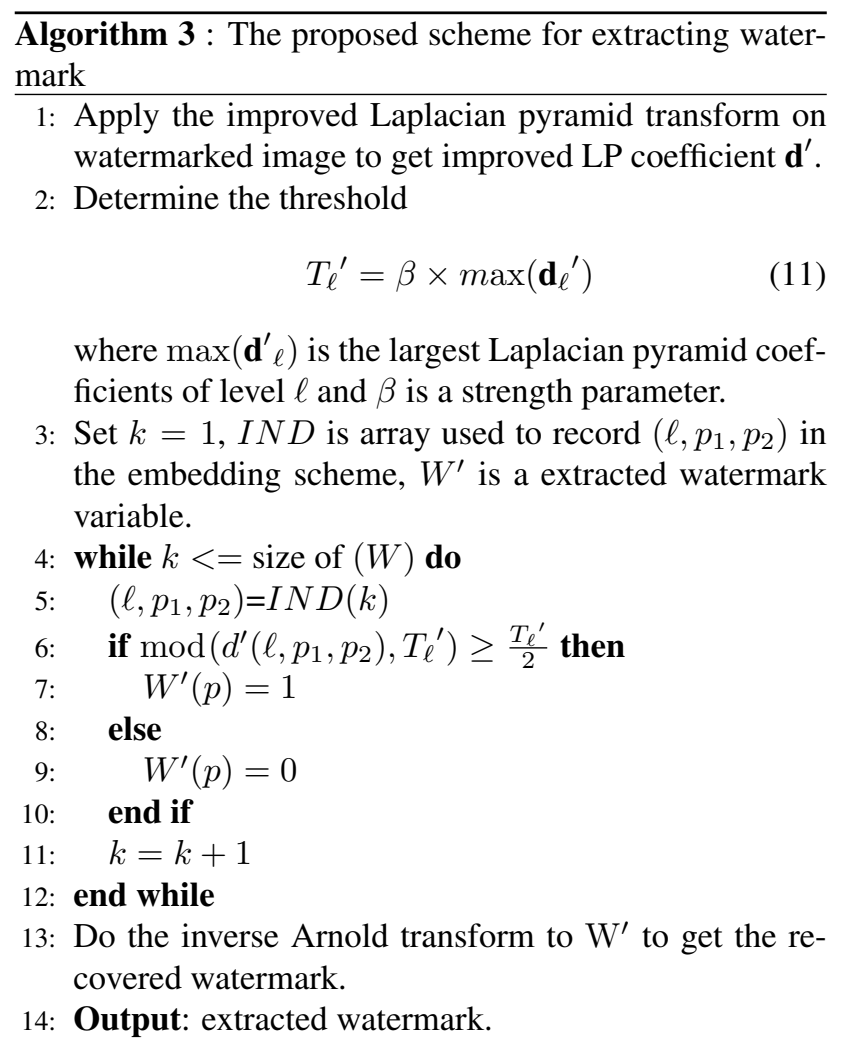

\section{Experiment results}

We carried out three experiments to evaluate the performance of the proposed watermarking scheme. In the experiments, we use the MATLAB R2013a as experimental platform on an Intel Core $i 5-2450 M C P U @ 2.50 \mathrm{GHz}$ personal computer with 4 GB RAM. The method in [17] has been improved in this paper by using combination the low frequency and the middle frequency to embed the watermark $\left(d_{5}, d_{4}\right.$ and $d_{3}$ as shown in Figure 5 are used). In addition, the watermark also has the size larger than that one in [17]. The host image is transformed into the frequency domain and is reconstructed to its spatial domain by using the improved Laplacian pyramid. As shown in $[17,19]$, the $9-7$ bi-orthogonal filters with five levels of pyramidal decomposition are used to decompose the host image. The value for $\beta$ is set to 0.2 as similar to those in [17]. The binary watermark after applying the Arnold function to enhance the security is embedded into the prediction errors of transformed domain.

\subsection{Experiment under JPEG lossy compression and Gaussian low pass filtering attacks}

In this experiment, the $512 \times 512$ pixels Lena gray image in Figure 6 (a) is used as a host image. Figure 6 (b) is used as a binary watermark whose size is $32 \times 32$ pixels. Figure 6 (c) and Figure 6 (d) are the watermarked image and recovered watermark without any attacks, respectively. 
Although in the proposed scheme, the low frequency subbands is exploited to embed the watermark, the PSNR between the host image and the watermarked image is quite high (PSNR $=47.016 \mathrm{~dB}$ as presented in Figure $6(\mathrm{c})$ ). In addition, it is very difficult to distinguish by human vision between the host image and the watermarked image. Furthermore, the proposed scheme can extract the watermark perfectly $(\mathrm{NC}=1)$ in the condition of no attacks.

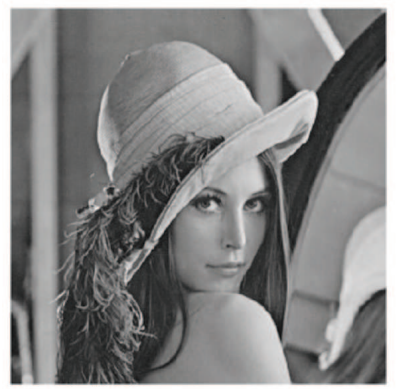

(a)

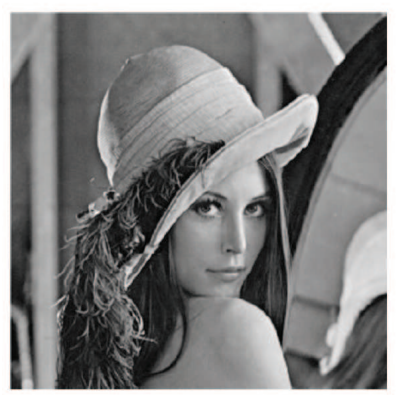

(c) $\operatorname{PSNR}=47.0160(\mathrm{~dB})$

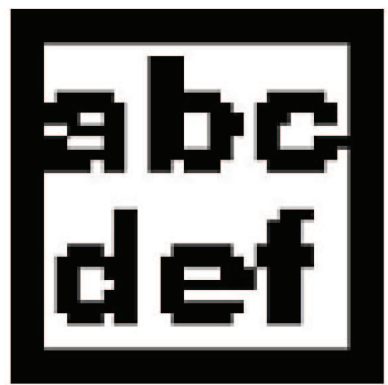

(b)

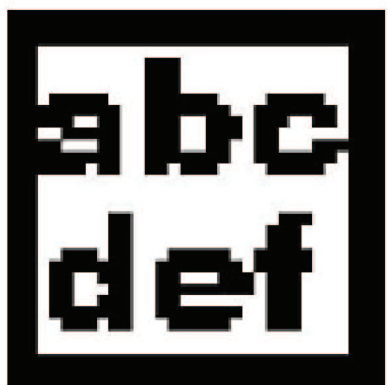

(d) $\mathrm{NC}=1$
Figure 6: (a) Lena host image, (b) original binary watermark, (c) watermarked image, (d) recovered watermark.

The robustness of the proposed scheme was tested by JPEG lossy compression and Gaussian low pass filtering. The experimental results are compared with those in $[11,12]$ as shown in Table 2, Table 3, Figure 7 and Figure 8 . The fidelity of the watermarked image after being attacked is evaluated by using the PSNR while the quality of the extracted watermark for some attacks is evaluated by the NC. The experimental results show that in terms of the robustness, the proposed scheme is superior to those in [11] under both JPEG lossy compression and Gaussian low pass filtering attacks. The robustness comparison between our proposed method with those in [12] is shown in Table 2. It can be seen from Table 2 that the NCs of the proposed scheme are higher than those by the method in [12] for almost all of the cases. Observing from Figure 7 and Figure 8 , we found that the quality of the extracted watermarks under JPEG lossy compression attacks and Gaussian low pass filtering attacks are very high. Figure 7 shows that the quality of the extracted watermarks is reduced as the compression ratio increases. As shown in Table 2, the PSNR of the watermarked image in the condition of no attack of the proposed scheme is comparable to those in $[11,12]$ and the
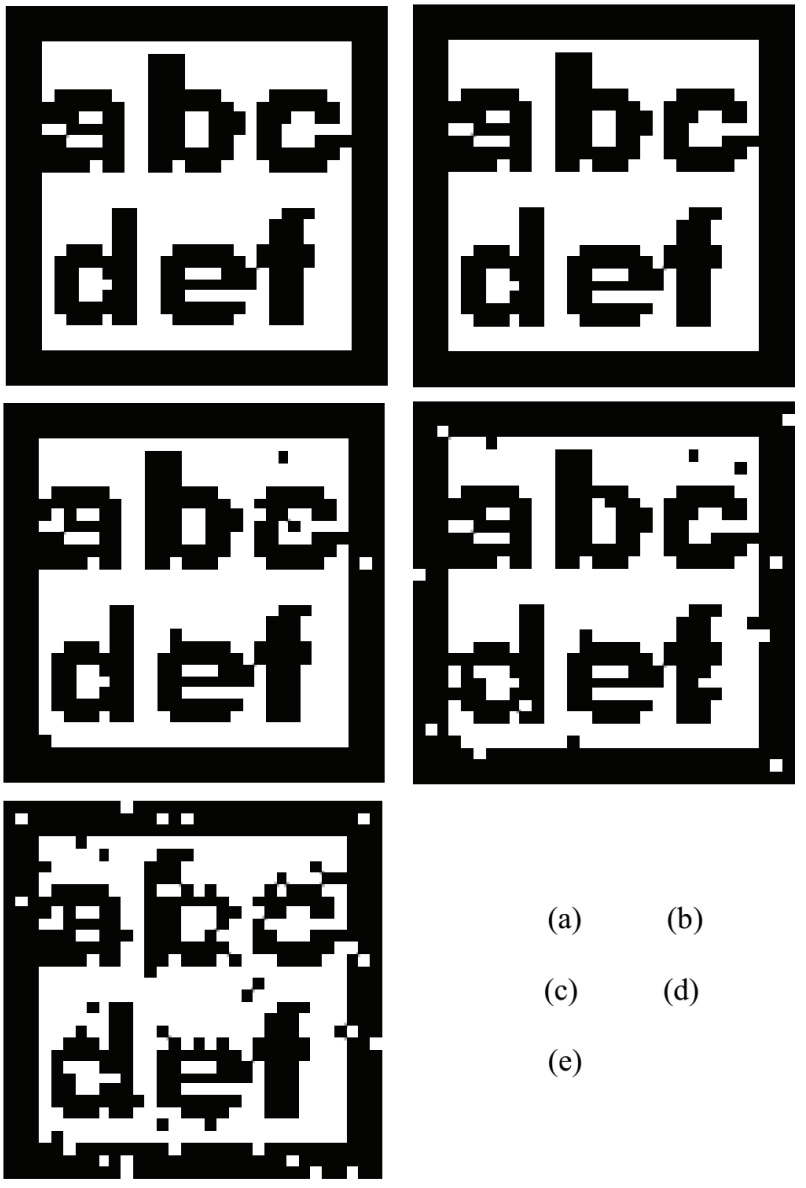

(a)

(b)

(c)

(d)

(e)

Figure 7: The extracted watermarks under JPEG lossy compression attacks:(a) no attack, (b) $Q=50$, (c) $Q=$ 30,(d) $Q=20$, (e) $Q=15$

quality of the watermarked image is acceptable. It is noted the methods in $[11,12]$ used the FDCT as the key transform in their watermarking schemes. By simulation, we also showed that the computational time of decomposition and reconstruction of our improved LP is lower than those of FDCT as listed in Table 5. This illustrates the computational efficiency of our proposed method.

\subsection{Experiment under intentional attacks}

In this experiment, we evaluate the performance of the proposed method in case of being attacked by intentional cutting $25 \%$ of the watermarked images. In cutting attacks, the attached pixels of the images are changed to black. Three images, namely, Baboon in Figure 9(a), Peppers in Figure 9(d) and Boat in Figure 9(g), are used as host images in this test. The binary watermark is the same as the watermark in Experiment 3.1 (i.e., Figure 6(b)). The PSNR is employed to measured the quality of watermarked image after being cut $25 \%$; The PSNR, NC and SSIM are employed to evaluate the robustness of the proposed method after being cutting intentional attacks. The results of this test are depicted in Figure 9 and are listed in Table 4. Although the water- 
Table 2: The comparison of the robustness against JPEG lossy compression of the proposed scheme with those in [11, 12].

\begin{tabular}{|c|c|c|c|c|c|c|}
\hline \multirow{2}{*}{ Attacks } & \multicolumn{2}{|c|}{ Reference [11] } & \multicolumn{2}{c|}{ Reference [12] } & \multicolumn{2}{c|}{ Proposed scheme } \\
\cline { 2 - 7 } & PSNR(dB) & NC & PSNR(dB) & NC & PSNR(dB) & NC \\
\hline Noattack & 50.12 & 1 & 60.80 & 1 & 47.02 & 1 \\
\hline $\mathrm{Q}=50$ & 36.33 & 0.998 & 24.97 & 0.991 & 35.31 & 1 \\
\hline $\mathrm{Q}=30$ & 35.17 & 0.995 & 24.89 & 0.974 & 34.03 & 0.991 \\
\hline $\mathrm{Q}=20$ & 33.51 & 0.971 & 24.67 & 0.947 & 32.47 & 0.972 \\
\hline $\mathrm{Q}=15$ & 32.30 & 0.945 & 24.48 & 0.911 & 31.81 & 0.933 \\
\hline
\end{tabular}

Table 3: The comparison of the robustness against Gaussian low pass filtering of the improved scheme with those in [11].

\begin{tabular}{|c|c|c|c|c|c|}
\hline$\sigma($ window $)$ & \multicolumn{2}{|c|}{ Proposed scheme } & \multicolumn{2}{c|}{ Reference [11] } \\
\hline & Recovered watermark & PSNR(dB) & NC & PSNR(dB) & NC \\
\hline $0.5(3)$ & Figure 8(a) & 39.6934 & 0.9977 & 40.8289 & 0.9914 \\
\hline $1.5(3)$ & Figure 8(b) & 32.0616 & 0.9748 & 32.4277 & 0.9704 \\
\hline $0.5(5)$ & Figure 8(c) & 39.7431 & 0.9954 & 40.8027 & 0.9914 \\
\hline $1.5(5)$ & Figure 8(d) & 29.4963 & 0.9537 & 29.9117 & 0.9113 \\
\hline $5.0(3)$ & Figure 8(e) & 31.5318 & 0.9725 & 28.7562 & 0.86582 \\
\hline
\end{tabular}

marked images are significantly destroyed by the cutting attacks which reveals by the low PSNR of the watermarked image, the watermark still can be extracted with the acceptable NC and high SSIM. In addition, the extracted watermark can be recognized by human vision. This result verifies the robustness of the proposed algorithm.

\subsection{Experiment for measuring the computational time}

This experiment evaluates the computational time of the proposed method using an improved LP transform (5 levels) as compared to other methods using FDCT [11, 12]. The host images are gray images of $512 \times 512$ pixels, including the Lena image in Figure 6 (a), the Elaine image in Figure $5\left(x_{0}\right)$ and the Peppers image in Figure 9 (d). The processing time of three methods is listed in Table 4. On average, the processing time of our improved LP is about 0.0572 seconds for both decomposition and reconstruction, and it is significantly lower those with methods with using FDCT.

\section{Conclusion}

In this work, we have presented an improved watermarking scheme using the LP coefficients in low and middle frequency sub-bands to embed binary watermark. The results of this research showed that the performance of the proposed algorithm in terms of invisibility and robustness is better than those using $2 D$ DWT and FDCT under JPEG lossy compression, Gaussian low pass filters, intentional cutting attacks. In addition, the proposed schemes require less computational time of embedding and extracting watermark than the other. In addition, the proposed method is blind watermarking and high security and, thus, the watermark is only deducted by the legal users.

\section{References}

[1] X. L. Liu, C. C. Lin, and S. M. Yuan, "Blind dual watermarking for color images' authentication and copyright protection," IEEE Transactions on Circuits and Systems for Video Technology, vol. 28, no. 5, pp. 1047-1055, 2018. https://doi.org/10.1109/tcsvt.2016. 2633878.

[2] S. Khalighi, P. Tirdad, and H. Rabiee, "A contourlet-based image watermarking scheme with high resistance to removal and geometrical attacks," EURASIP Journal on Advances in Signal Processing, vol. 2010, no. 21, pp. 1-13, 2010. http://dx.doi.org/10.1155/2010/540723.

[3] B. L. Gunjal and R. R. Manthalkar, "An overview of transform domain robust digital image watermarking algorithms," in Journal of Emerging trends in Computing and Information Science, 2010, pp. 37-42. http://www.cisjournal.org/Download_pdf_2_ 5.aspx.

[4] J. Liu, Y. Xu, S. Wang, and C. Zhu, "Complex wavelet-domain image watermarking algorithm using L1-Norm function-based quantization," Circuits, Systems, and Signal Processing, pp. 1-19, 2017. https://doi.org/10.1007/s00034-017-0607-5. 
Table 4: The empirical results of invisibility and robustness of proposed against $25 \%$ cutting attacks.

\begin{tabular}{|c|c|c|c|c|}
\hline \multirow{2}{*}{ Host image } & Attacked image & \multicolumn{3}{|c|}{ Recovered watermark } \\
\cline { 2 - 5 } & PSNR $(d B)$ & NC & PSNR $(d B)$ & SSIM \\
\hline Baboon & 8.7030 & 0.7092 & 6.1760 & 0.9926 \\
\hline Peppers & 9.5256 & 0.7555 & 7.2700 & 0.9912 \\
\hline Boat & 10.5776 & 0.8123 & 8.1164 & 0.9953 \\
\hline Average & 9.6021 & 0.7590 & 7.1875 & 0.9930 \\
\hline
\end{tabular}

Table 5: Comparison the processing times of 2 transforms: FDCT(wrapping) and Improved LP.

\begin{tabular}{|c|c|c|c|c|}
\hline \multirow{2}{*}{ Host image } & \multicolumn{2}{|c|}{ FDCT(wrapping) } & \multicolumn{2}{c|}{ Improved LP } \\
\cline { 2 - 5 } & Decomposition(s) & Reconstruction(s) & Decomposition(s) & Reconstruction(s) \\
\hline Lena & 0.4212 & 0.6864 & 0.0468 & 0.0312 \\
\hline Elain & 0.4836 & 0.5304 & 0.0468 & 0.0780 \\
\hline Peppers & 0.3744 & 0.5460 & 0.0780 & 0.0624 \\
\hline Average & 0.4264 & 0.5876 & 0.0572 & 0.0572 \\
\hline
\end{tabular}

[5] L.-Y. Hsu and H.-T. Hu, "Robust blind image watermarking using crisscross inter-block prediction in the DCT domain," Journal of Visual Communication and Image Representation, vol. 46, no. Supplement C, pp. 33 - 47, 2017. https://doi.org/10.1016/j.jvcir.2017.03.009.

[6] V. M. Potdar, S. Han, and E. Chang, "A survey of digital image watermarking techniques," in INDIN '05. 2005 3rd IEEE International Conference on Industrial Informatics, 2005., 2005, pp. 709-716. https://doi.org/10.1109/indin.2005.1560462.

[7] P. Tao and A. M. Eskicioglu, "A robust multiple watermarking scheme in the discrete wavelet transform domain," in Internet Multimedia Management Systems V, vol. 5601, 2004, pp. 133-144. http://dx.doi.org/10.1117/12.569641.

[8] S. P. Singh, P. Rawat, and S. Agrawal, "A robust watermarking approach using DCT-DWT," International Journal of Emerging Technology and Advanced Engineering, vol. 2, pp. 300-305, 2012. https://ijetae. com/files/Volume2Issue8/IJETAE_0812_52.pdf.

[9] N. S. Narawade and R. D.KanphadeTaiyue, "DCT based robust reversible watermarking for geometric attack," International Journal of Emerging Trends and Technology in Computer Science (IJETTCS), vol. 1, pp. 27-32, 2012. https://www.ijettcs.org/ Volume1Issue2/IJETTCS-2012-07-25-025.pdf.

[10] W. Bender, W. Butera, D. Gruhl, R. Hwang, F. J. Paiz, and S. Pogreb, "Applications for data hiding," IBM Systems Journal, vol. 39, no. 3.4, pp. 547-568, 2000. https://doi.org/10.1147/sj.393.0547.
[11] Z. y. Zhang, W. Huang, J. 1. Zhang, H. y. Yu, and Y. j. Lu, "Digital image watermark algorithm in the curvelet domain," in 2006 International Conference on Intelligent Information Hiding and Multimedia, 2006, pp. 105-108. https://doi.org/10.1109/iih-msp. 2006.264965 .

[12] H. P. J. Xu and J. Zhao, "Digital image watermarking algorithm based on fast curvelet transform," Journal of Software Engineering and Applications, vol. 3, no. 10, pp. 939-943, 2010. https://doi.org/10.4236/ jsea.2010.310111.

[13] S. C. Nguyen, K. H. Ha, and H. M. Nguyen, "An improved image watermarking scheme using selective curvelet scales," in 2015 International Conference on Advanced Technologies for Communications (ATC), 2015, pp. 445-450. https://doi.org/10.1109/atc.2015.7388369.

[14] P. Burt and E. Adelson, "The laplacian pyramid as a compact image code," IEEE Transactions on Communications, vol. 31, no. 4, pp. 532-540, 1983. https://doi.org/10.1109/tcom.1983.1095851.

[15] M. N. Do and M. Vetterli, "Framing pyramids," IEEE Transactions on Signal Processing, vol. 51, no. 9, pp. 2329-2342, 2003. https://doi.org/10.1109/tsp.2003. 815389.

[16] S. C. Nguyen, H. H. Kha, and H. M. Nguyen, "A new image watermarking scheme using contourlet transforms," in The 3nd International Conference on Information Technology, Computer, And Electrical Engineering ( ICITACEE 2016 ), 2016, pp. 283-288. https://doi.org/10.1109/icitacee.2016.7892456. 

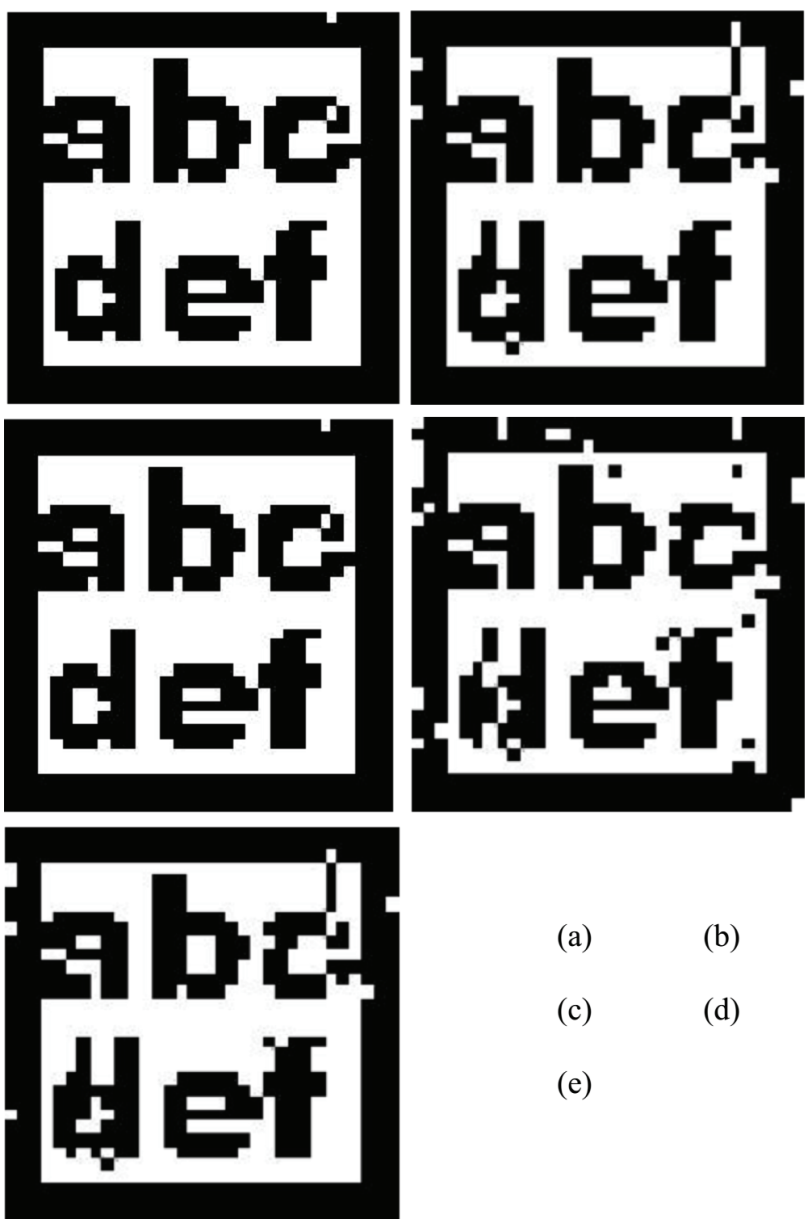

(a)

(c)

(b)

(d)

(e)
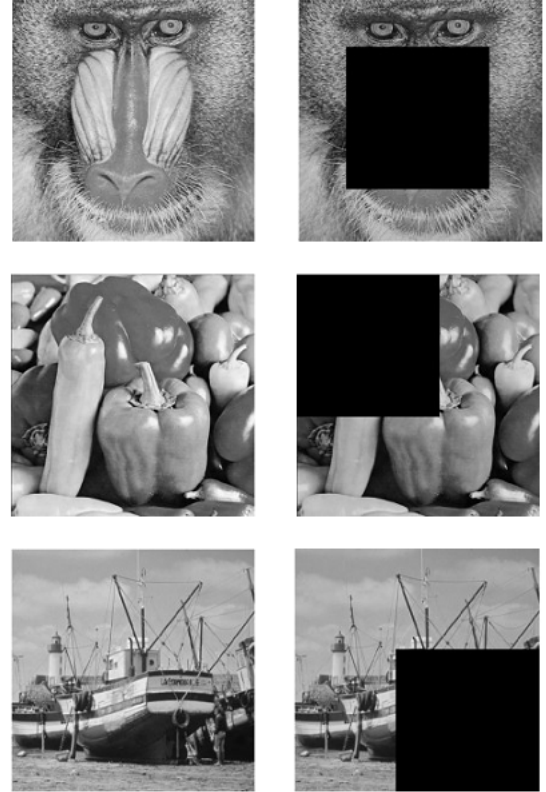

(b)

(c)

(d) (e)

(f)

(g) (h)

(k)
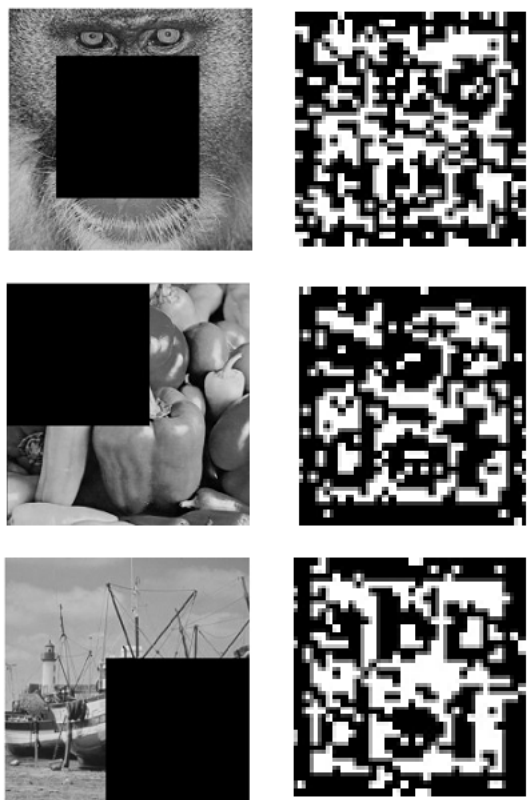
$\sigma($ window $)=1.5(5)$, (e) $\sigma($ window $)=5.0(3)$.

[17] S. C. Nguyen, H. H. Kha, and H. M. Nguyen, "An efficient image watermarking scheme using the laplacian pyramid based on projection," in 2017 International Conference on Recent Advances in Signal Processing, Telecommunications Computing (SigTelCom), 2017, pp. 103-108. https://doi.org/10. 1109/sigtelcom.2017.7849804.

[18] Z. Wang, A. C. Bovik, H. R. Sheikh, and E. P. Simoncelli, "Image quality assessment: from error visibility to structural similarity," IEEE Transactions on Image Processing, vol. 13, no. 4, pp. 600-612, 2004. https://doi.org/10.1109/tip.2003.819861.

[19] D. D. Y. Po and M. N. Do, "Directional multiscale modeling of images using the contourlet transform," IEEE Transactions on Image Processing, vol. 15, no. 6, pp. 1610-1620, 2006. https://doi.org/10.1109/ ssp.2003.1289394.

Figure 9: cropping 25\% attacks, (a) The Baboon host image, (d) The Peppers host image, (g) The Boat host image; (b) The Baboon watermarked image, (e) The Peppers watermarked image, (h) The Boat watermarked image; (c) recovered watermark from the Baboon watermarked image, (f) recovered watermark from the Peppers watermarked image, $(\mathrm{k})$ recovered watermark from the Boat watermarked image. 